\title{
Evapotranspiração máxima do pimentão cultivado em estufa plástica em função de variáveis fenométricas e meteorológicas ${ }^{1}$
}

Carina R. Pivetta ${ }^{2}$, Arno B. Heldwein ${ }^{2}$, Ivan C. Maldaner², Sidinei Z. Radons², Ivonete F. Tazzo² \& Dionéia D. Lucas ${ }^{2}$

\begin{abstract}
RESUMO
O bjetivou-se, neste trabalho, estimar a evapotranspiração máxima (ETm) do pimentão, em função da evaporação medida no evaporímetro de Piche (Epi) exposto à radiação solar, com e sem a inclusão de variáveis meteorológicas e fenométricas pontuais de fácil mensuração. Em uma estufa plástica de $240 \mathrm{~m}^{2}$ se realizaram medidas da ETm (em lisímetros de drenagem), Epi, temperatura do ar, déficit de saturação do ar (Di), índice de área foliar (IAF), altura de plantas (AP) e número de folhas (NF). Ajustaram-se modelos para estimar a ETm com os dados coletados selecionando-se apenas os que apresentaram parâmetros angulares significativos pelo teste ta $5 \%$ de probabilidade de erro e coeficiente de determinação $\left(R^{2}\right)>0,80.0$ btiveram-se os mel hores modelos para a estimativa da ETm com os valores da Epi associados a um parâmetro de crescimento das plantas, como IAF, NF e AP. Foi possível estimar a ETm do pimentão com precisão aceitável ( $R^{2}$ entre 0,81 e 0,91), para fins de irrigação sob estufa, por meio de modelos que incluíram a Epi e pelo menos uma variável fenométrica, preferencialmente a AP. A inclusão de Di medido às $9 \mathrm{~h}$ nos modelos em que se incluíram a Epi e AP ou NF ou IAF, melhorou a estimativa da ETm.
\end{abstract}

Palavras-chave: Capsicum annuum, ambiente protegido, piche

\section{Maximum evapotranspiration of sweet pepper grown in plastic greenhouse based upon meteorological and fenometrical variables}

\begin{abstract}
The objective of this study was to estimate maximum evapotranspiration (ETm) of sweet pepper inside a plastic greenhouse as a function of evaporation measured with "Piche" evaporimeter (Epi) exposed to solar radiation, with and without easily measured meteorological and phenologic variables. The experiment was carried out inside a $240 \mathrm{~m}^{2}$ plastic greenhouse. Daily ETm (measured with drainage lysimeters), Epi, air temperature, vapor pressure deficit (Di), leaf area index (LAI), plant height (PH), and leaf number $(\mathrm{LN})$ were measured. Models were fitted to estimate ETm using data collected on even days of the experimental period, selecting only models with parameters significant at $5 \%$ by t test and coefficient of determination $\left(R^{2}\right)>0.80$. The best models for estimating ETm were obtained with Epi values, associated with a plant growth parameter, such as LAI, LN and PH. It was possible to estimate ETm of sweet pepper crop with acceptable accuracy ( $R^{2}$ between 0.81 and 0.91 ) to perform irrigation inside plastic greenhouse using models that take into account Epi and at least one plant growth parameter, however models with $\mathrm{PH}$ should be preferred. Including Di measured at $9 \mathrm{~h}$ in the models with Epi and PH or NL or LAI improved ETm estimates.
\end{abstract}

Key words: Capsicum annuum, greenhouse, piche

\footnotetext{
1 Parte da Dissertação de Mestrado em Produção Vegetal da primeira autora pela UFSM

${ }^{2}$ Departamento de Fitotecnia/UFSM. Avenida Roraima n.1000, Prédio 77, Cidade Universitária, Bairro Camobi, CEP 97105-900, Santa Maria, RS. Fone: (55) 3220-8179. E-mail: carina.pivetta@gmail.com; heldwein@ccr.ufsm.br; ivan_maldaner@yahoo.com.br; sidineiradons@yahoo.com.br; ivonetetazzo@yahoo.com.br; dio.pitol@gmail.com
} 


\section{INTRODUÇãO}

O pimentão (Capsicum annuum) é uma das cinco culturas com maior área em cultivo protegido, tanto no Brasil como em diversos países devido à grande produtividade e qualidade dos frutos nessas condições (Lorentz et al., 2002) porém, para assegurar essas vantagens é preciso adequar o manejo ao ambiente protegido principalmente quanto à suplementação hídrica pois o pimentão é muito exigente em água e necessita que a irrigação seja feita com base em critérios técnicos para evitar danos.

A evapotranspiração compreende simultaneamente a evaporação e a transpiração, processos controlados pelo suprimento de água às plantas e pela disponibilidade de energia resultante da interação com as variáveis meteorológicas que condicionam a demanda atmosférica (Pereira et al., 1997). Ocorre naturalmente como forma de dissipar energia e manter o metabolismo para o crescimento e desenvolvimento do vegetal.

Conforme resultados obtidos por Dalmago (2001) com a cultura do pimentão, os elementos meteorológicos mais importantes e que melhor determinam a evapotranspiração sob estufas, são a radiação solar global incidente ou saldo de radiação e o déficit de saturação do ar.

A cobertura plástica é a maior responsável pelas alterações meteorológicas que ocorrem dentro de uma estufa, possibilita ajustar o ambiente às plantas e estender o período de produção para épocas do ano e mesmo regiões antes inaptas à agricultura (Andriolo, 1999), sobretudo para os cultivos na entressafra, ampliando o rendimento econômico dos produtores (Araújo et al., 2009). Isto se aplica especialmente aos produtores da região Sul do País (Valandro et al., 2007), uma vez que nas regiões mais frias o cultivo de muitas das espécies hortícolas fica restrito à época mais quente do ano em função de suas exigências térmicas.

Basicamente, o que ocorre dentro da estufa é a atenuação das variações extremas dos elementos meteorológicos desfavoráveis a essas culturas no ambiente externo (Vasquéz et al., 2005), como a redução da radiação solar incidente (Buriol et al., 2005; Beckmann et al., 2006), velocidade do vento (Cardoso et al., 2008; Chavarria et al., 2008), aumento da temperatura do ar, do solo e da umidade relativa do ar (Heldwein et al., 2001; Reis et al., 2009).

Dentro da estufa se forma um microclima que, geralmente, atua na redução da magnitude da evapotranspiração que pode ser de até 70\% (Dalmago et al., 2006) e, consequentemente, afeta o consumo de água pelas plantas, razão pela qual é indispensável reformular o manejo da irrigação nesses ambientes, em que a precipitação é ausente e toda a água necessária para o desenvolvimento das plantas precisa ser fornecida e, portanto, pode ser controlada nos níveis ideais para a cultura.

A irrigação consome grande quantidade de água e deve ser realizada com alta eficiência para minimizar perdas por percolação e poluição ambiental (Bonachella et al., 2006) de acordo com a evapotranspiração calculada para cada cultura, informação que ainda é escassa (Orgaz et al., 2005).
Uma hipótese plausível para determinar as necessidades hídricas dos cultivos é estabelecer relações entre a evapotranspiração máxima da cultura e alguns parâmetros de crescimento das plantas, como o índice de área foliar, número de folhas e altura de plantas. Por outro lado, pode-se não só utilizar elementos meteorológicos mas também medi-los interna ou externamente à estufa; isto pode ser factível mediante a modelagem, a qual permite ampliar a viabilidade dos resultados para uma região de abrangência muito maior desde que envolvam elementos meteorológicos que têm representatividade para áreas relativamente grandes.

Na literatura são encontradas referências à relação da evapotranspiração com a radiação solar e a temperatura diária, como as opções mais utilizadas para estimar as necessidades hídricas dos cultivos em estufa (Bonachella et al., 2006).

Para buscar alternativas que visem simplificar os modelos existentes e reduzir os custos de medição de variáveis, vemse desenvolvendo estudos referentes à utilização de evaporímetros, que apresentam as vantagens do fácil manejo e do baixo custo (Pivetta, 2007); alguns tipos podem ser mantidos acima do nível do dossel e, consequentemente, evitar o efeito do sombreamento pela cultura, além de ocuparem pequeno espaço.

Uma alternativa simples para quantificar a água a ser irrigada pode ser a utilização da evaporação medida no evaporímetro de Piche em modelos que incluem pelo menos uma variável que represente o crescimento da planta (Heldwein et al., 2001), levando-se em consideração que, para tal, o evaporímetro deve ser instalado exposto a radiação solar, de forma similar às folhas superiores das plantas e a superfície de evaporação deve apresentar algumas propriedades físicas similares às folhas.

Objetivou-se, neste estudo, obter modelos para estimar a evapotranspiração máxima do pimentão cultivado em estufa plástica, em função da evaporação medida no evaporímetro de Piche adicionada de variáveis fenométricas e/ou meteorológicas.

\section{MATERIAL E MÉTODOS}

Dois experimentos foram conduzidos em estufa plástica, na área experimental do Departamento de Fitotecnia da Universidade Federal de Santa Maria, RS, Brasil (latitude: $29^{\circ} 43^{\prime} 23^{\prime \prime}$ $\mathrm{S}$, longitude: $53^{\circ} 43^{\prime} 15^{\prime \prime} \mathrm{W}$ e altitude: $95 \mathrm{~m}$ ). O clima da região, segundo a classificação de Köppen, é do tipo Cfa.

A estufa plástica possuía $24 \mathrm{~m}$ de comprimento por $10 \mathrm{~m}$ de largura $\left(240 \mathrm{~m}^{2}\right), 2,0 \mathrm{~m}$ de pé-direito, $3,5 \mathrm{~m}$ de altura na parte central e era orientada no sentido Norte-Sul; sua cobertura era em forma de arco, modelo arco-pampeana e coberta por polietileno de baixa densidade transparente com $100 \mu \mathrm{m}$ de espessura.

O manejo de abertura e fechamento da estufa foi realizado conforme as condições meteorológicas no decorrer do dia. Em dias de tempo bom, não frios, a abertura foi realizada ao amanhecer e o fechamento ao entardecer; nos dias frios, com previsão de geada, a estufa foi fechada nas primeiras horas da tarde para se conseguir maior armazenamento de calor em 
seu interior. Nos dias com precipitação intensa ou ventos fortes, principalmente os do quadrante Norte, somente as portas frontais da estufa eram mantidas abertas.

Realizou-se a adubação de acordo com os resultados da análise do solo, para a camada de $0-20 \mathrm{~cm}$, seguindo-se as recomendações oficiais da CQFS (2004) com a incorporação do adubo realizada no momento da construção dos camalhões, os quais foram recobertos com polietileno de baixa densidade opaco preto.

As semeaduras do pimentão, Capsicum annuum L., híbrido "Vidi", foram realizadas em 16/06/2005 e 20/06/2006, em bandejas de 128 células preenchidas com substrato comercial; o transplante foi efetuado em 09/08/2005 e em 13/09/2006 e os experimentos finalizados em 18/12/2005 e 07/12/2006, respectivamente.

Fez-se a determinação da evapotranspiração máxima (ETm, $\mathrm{mm} \mathrm{dia}{ }^{-1}$ ) em três repetições de lisímetros de drenagem com substrato (LS), construídos conforme Valandro et al. (1999). Em cada LS foram cultivadas cinco plantas no interior de sacolas plásticas preenchidas com oito litros de substrato comercial que estavam acondicionadas em uma calha de PVC com 1,5 m de comprimento e impermeabilizadas com o mesmo PEBD que recobriu os camalhões.

A ETm de cada dia foi contabilizada pela soma das diferenças de volume de água irrigada e drenada em cada uma das duas irrigações diárias. O fornecimento de água em cada irrigação foi interrompido ao se iniciar a drenagem do excedente, que era recolhido em um recipiente na extremidade do lisímetro.

A evaporação nos evaporímetros de Piche (Epi, mm dia ${ }^{-1}$ ) foi medida diariamente, entre 7 e $8 \mathrm{~h}$ da manhã, em três evaporímetros instalados fora do abrigo meteorológico, sobre cada um dos LS, a uma altura de 1,5 m do solo para que não fossem sombreados pelo dossel de plantas. A Epi diária foi calculada pela diferença de nível da água medida no menisco inferior do evaporímetro, em dias subsequentes. O disco de papel filtro que constituía a superfície evaporante na extremidade inferior do evaporímetro, com uma área de $13,2 \mathrm{~cm}^{2}$, foi substituído a cada sete dias ou em caso de dano mecânico.

Realizaram-se medições fenométricas semanais a fim de determinar o índice de área foliar (IAF), altura das plantas (AP) e número de folhas (NF) por planta. Para determinar a área foliar (AF), medidas lineares do comprimento (C), em centímetros, de todas as folhas de duas plantas marcadas aleatoriamente em cada lisímetro, foram realizadas, enquanto a $\mathrm{AF}$ foi calculada pela seguinte função exponencial (Dalmago, 2001):

$$
\mathrm{AF}=0,464 * \mathrm{C}^{1,84}
$$

A partir dos dados de AF determinou-se o IAF diário, por interpolação, com o ajuste de equações em função do número de dias após o transplante (DAT).

Além da Epi foram medidas, também, outras variáveis meteorológicas no interior da estufa, como a radiação solar, temperatura do ar, umidade relativa do ar e déficit de saturação do ar. A temperatura, a umidade relativa do ar e o déficit de saturação do ar foram obtidos por dois pares psicrométricos, constituídos de dois sensores Pt-100 de resistência elétrica de platina, isto é, um seco e outro úmido, instalados a 1,5 m do solo no interior de um abrigo meteorológico com ventilação natural. A radiação solar foi medida por um tubo solarímetro, instalado a uma altura de 2,5 m do solo próximo ao teto da estufa. Esses equipamentos foram instalados na área central da estufa e conectados a um sistema automático de aquisição de dados (datalogger) alimentado por uma bateria de $12 \mathrm{~V}$ e com registro contínuo dos dados, a cada $10 \mathrm{~min}$.

Semanalmente, realizou-se a transferência dos dados para processamento obtendo-se os valores diários de temperatura máxima $\left(\mathrm{Tmax},{ }^{\circ} \mathrm{C}\right)$, temperatura mínima $\left(\mathrm{Tmin},{ }^{\circ} \mathrm{C}\right)$, temperatura média do ar (Tmi, $\left.{ }^{\circ} \mathrm{C}\right)$, amplitude térmica diária do ar (AT, $\left.{ }^{\circ} \mathrm{C}\right)$ e temperatura do ar, às $9 \mathrm{~h}\left(\mathrm{~T} 9,{ }^{\circ} \mathrm{C}\right)$, às $15 \mathrm{~h}\left(\mathrm{~T} 15,{ }^{\circ} \mathrm{C}\right)$ e às $21 \mathrm{~h}\left(\mathrm{~T} 21,{ }^{\circ} \mathrm{C}\right)$, umidade relativa do ar às $9 \mathrm{~h}$ (UR9, \%), às 15 $\mathrm{h}$ (UR15, \%), às $21 \mathrm{~h}$ (UR21, \%), umidade relativa média diária do ar (URmi, \%), déficit de saturação do ar às 9 h (D9, hPa), às $15 \mathrm{~h}(\mathrm{D} 15, \mathrm{hPa})$ e médio diário $(\mathrm{Di}, \mathrm{hPa})$ e radiação solar global incidente [Rgi; $\mathrm{MJ} \mathrm{m}^{-2}\left(10 \mathrm{~min}^{-1}\right.$ ]. Os dados meteorológicos do ambiente externo à estufa plástica foram obtidos de uma estação meteorológica situada acerca de $100 \mathrm{~m}$ do local do experimento.

Estimou-se a ETm através de modelos de regressão simples e múltipla trabalhando-se com o banco de dados coletado no primeiro experimento, na primavera de 2005, para o que se consideraram, além da ETm, a ETm por unidade de raiz quadrada de índice de área foliar $\left(\mathrm{ETm} \mathrm{IAF}^{-0,5}\right)$ de altura de plantas $\left(\mathrm{ETm}_{\mathrm{AP}} \mathrm{AP}^{-0,5}\right)$ e de número de folhas $\left(\mathrm{ETm} \mathrm{NF}^{-0,5}\right)$ e suas relações com as variáveis fenométricas e meteorológicas.

Em todos os modelos foi incluída a Epi, além de um parâmetro que representasse a condição de crescimento da planta, como o IAF, a AP e o NF. Em alguns modelos também foram incluídas variáveis meteorológicas medidas em um horário fixo no interior da estufa.

Os dados foram processados em planilhas excel e os modelos de estimativa da ETm gerados por meio de um software específico, o programa de linguagem SAS (Statistical Analysis System), usado também para se proceder às análises estatísticas.

O primeiro critério utilizado para selecionar e enumerar os melhores modelos de regressão foi o coeficiente de determinação $\left(\mathrm{R}^{2}\right)$, o qual deveria apresentar parâmetros angulares significativos pelo teste $\mathrm{t}$ a $5 \%$ de probabilidade e ser maior do que 0,80 .

Os modelos gerados com os dados meteorológicos da primavera de 2005 foram selecionados e testados com os dados coletados no segundo experimento, na primavera de 2006. A partir da comparação entre os valores de ETm medida em 2005 e a estimada pelos modelos com os dados de 2006, procedeuse à avaliação do desempenho dos modelos que foi realizada conforme Camargo \& Sentelhas (1997), considerando-se a raiz do quadrado médio do erro (RMSE), $\mathrm{R}^{2}, \mathrm{r}$, d e c (Heldwein et al., 2004).

\section{RESULTADOS E DISCUSSÃO}

Verificou-se a relação dos valores diários da ETm com a radiação solar global, a temperatura média do ar e o déficit de 
saturação médio do ar medidos no interior da estufa (Figura 1). Esses resultados estão de acordo com os obtidos por Dalmago et al. (2006) que concluíram que a temperatura do ar, o saldo de radiação e o déficit de saturação do ar medidos no interior da estufa são as variáveis meteorológicas que apresentam maior relação com a ETm do pimentão em ambiente protegido. Righi et al. (2002) também verificaram, no período de outono-inverno, que o déficit de saturação do ar foi a variável meteorológica que apresentou melhor relação com a transpiração do tomateiro, com coeficiente de determinação de 0,92 .

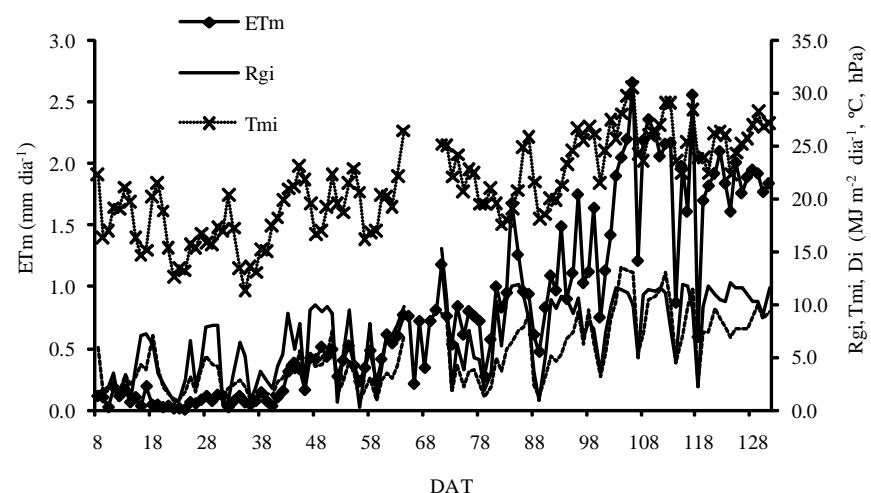

Figura 1. Evapotranspiração máxima (ETm, mm dia-1) associada à radiação solar global (Rgi, MJ m-2 $\left.\mathrm{dia}^{-1}\right)$, temperatura média do ar $\left(\mathrm{Tmi}^{\circ}{ }^{\circ} \mathrm{C}\right)$ e ao déficit de saturação médio do ar (Di, hPa), medidos no interior da estufa em função dos dias após o transplante (DAT) para o pimentão cultivado em estufa plástica na primavera de 2005

Ao longo dos 131 dias do período experimental, a ETm acumulou $101,5 \mathrm{~mm}$ e uma média de $0,81 \mathrm{~mm} \mathrm{dia}^{-1}$; neste mesmo período, a Epi totalizou $353,8 \mathrm{~mm}$ com a média de $2,8 \mathrm{~mm} \mathrm{dia}^{-1}$. Até os 60 DAT, a ETm se manteve inferior a $0,5 \mathrm{~mm} \mathrm{dia}{ }^{-1}$ em virtude, quiçá, do reduzido IAF das plantas. Referidos valores são muito inferiores aos da Epi que, na maioria dos dias, foi superior a 2,0 $\mathrm{mm} \mathrm{dia}^{-1}$ (Figura 3A).

O valor médio diário da ETm encontrada para o pimentão é considerado baixo para o período da primavera, em que as condições meteorológicas tendem a elevar a demanda hídrica atmosférica e a evapotranspiração. Contudo, a cobertura plástica altera os elementos meteorológicos no interior da estufa, reduzindo a radiação solar global, a velocidade do vento, o déficit de saturação do ar e, consequentemente, a demanda hídrica atmosférica (Dalmago et al., 2003) e, por esta razão, a ETm é menor em relação aos cultivos no ambiente externo, condição esta verificada, realmente, com um aumento gradativo da densidade do fluxo de radiação solar global incidente no ambiente externo à estufa (Rge), alcançando cerca de 24 $\mathrm{MJ} \mathrm{m}{ }^{-2} \mathrm{dia}^{-1}$ no final do experimento (Figura 2A).

Dalmago et al. (2003) encontraram uma média geral de 1,21 $\mathrm{mm}$ dia $^{-1}$ para a ETm do pimentão também cultivado em estufa plástica. Tazzo et al. (2004) mencionaram uma ETm de 1,3 $\mathrm{mm} \mathrm{dia}{ }^{-1}$ para a primavera de 2000 e $1,5 \mathrm{~mm} \mathrm{dia}^{-1}$ em 2001; entretanto, Tazzo et al. (2004) citam que a causa para a diminuição na ETm pode estar relacionada à ocorrência de perío-

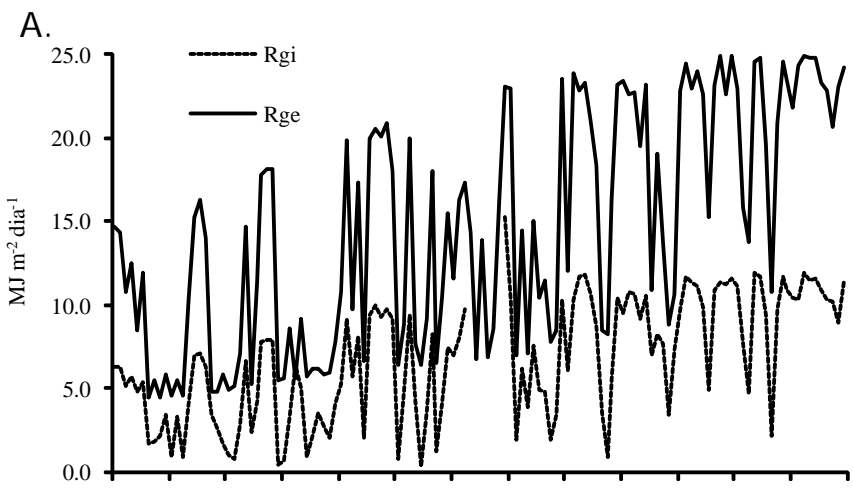

B.

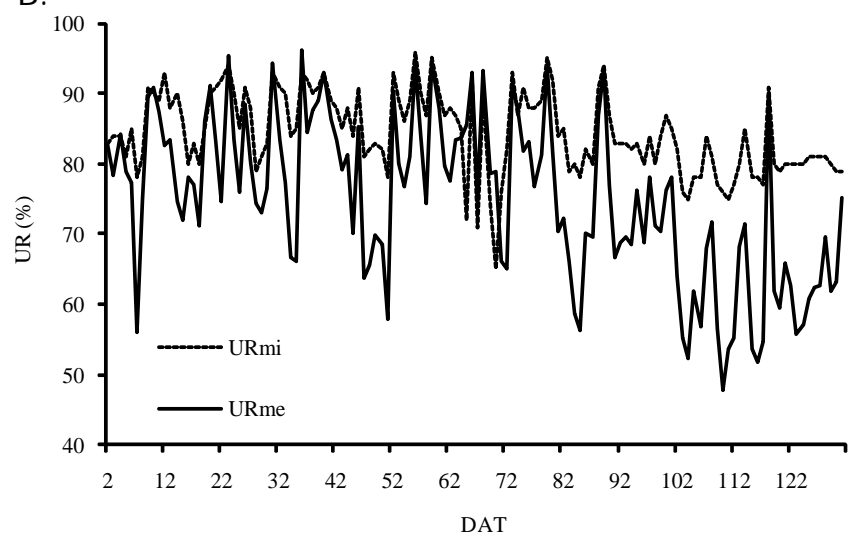

Figura 2. Densidade do fluxo de radiaç ão solar global incidente (A) no ambiente interno (Rgi, MJ m${ }^{-2}$ dia-1) $^{-1}$ e externo (Rge, $M J ~^{-2}$ dia $^{-1}$ ) (B) umidade relativa do ar interna (URmi, \%) e externa (URme, \%) em função dos dias após o transplante (DAT) na primavera de 2005

dos chuvosos e prolongados que reduzem a demanda hídrica atmosférica devido à baixa insolação e ao maior tempo em que a estufa permanece fechada. Esta condição de fato ocorreu na primavera de 2005, a qual se caracterizou por ser muito chuvosa e pode ter sido decisiva para a maior influência do déficit de saturação do ar na redução da ETm.

Atrelado a esta condição e contribuindo para a redução na média diária da ETm, se acha a umidade relativa do ar, pela influência que exerce na redução do déficit de saturação do ar. As variações na URmi são determinadas principalmente pelo manejo de abertura e fechamento das cortinas e portas da estufa. Aos 22, 23, 24, 26, 27, 31, 32, 33, 36, 38, 40, 46, 52, 56, 89 e 118 DAT a estufa permaneceu somente com as portas frontais abertas, ou seja, nos dias com precipitação ou ventos fortes as cortinas laterais foram fechadas, condição que determinou a redução do déficit de saturação do ar e da ETm (Figura 1).

Na maioria dos dias, os maiores valores de umidade relativa do ar ocorreram no interior da estufa (URmi) (Figura 2B), sobretudo nos dias em que a URme foi menor, o que ocorreu com maior frequência após os 77 DAT. Isto provavelmente está relacionado com a maior ocupação do espaço aéreo pelas plantas dentro da estufa, reduzindo a circulação de ar e menor contribuição da transpiração das plantas.

Nos dias mais úmidos, em que a URme era superior a 90\%, os valores internos e externos tenderam a ser semelhantes (Figura 2B). Os maiores valores de umidade relativa do ar foram de $96 \%$ no interior e de $94 \%$ no exterior da estufa, ocor- 


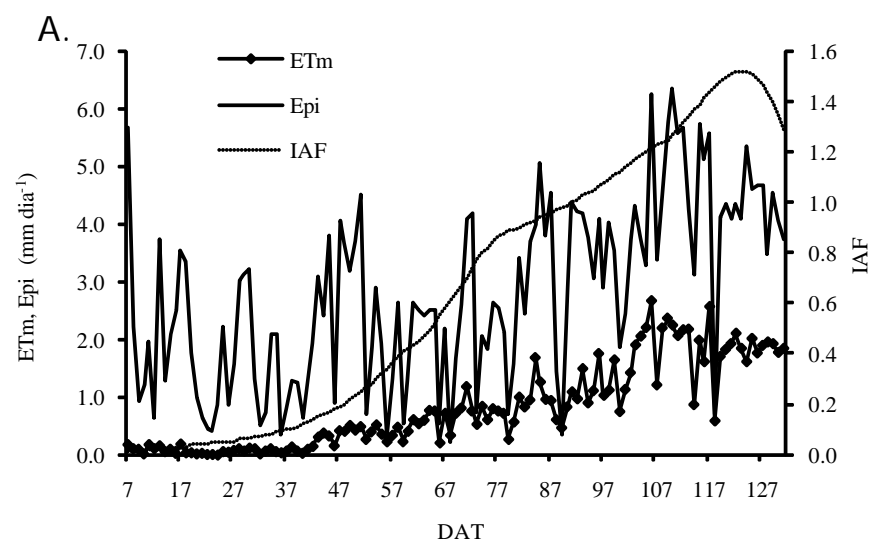

B.

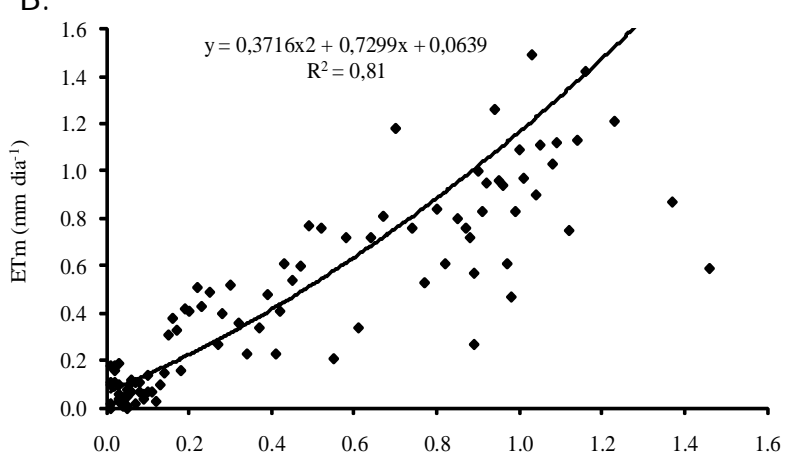

IAF

Figura 3. Evapotranspiração máxima diária (ETm, $\left.\mathrm{mm}^{\mathrm{d}} \mathrm{dia}^{-1}\right)$, evaporação medida no evaporímetro de Piche (Epi, $\mathrm{mm}_{\mathrm{m}}$ dia-1$^{-1}$ ) e índice de área foliar (IAF) em função do número de dias após o transplante (DAT) (A) e regressão entre ETm e o IAF (B), para o pimentão cultivado na primavera, em estufa plástica

ridos aos 56 DAT (04/10/2005), data em que ocorreu uma precipitação de $78 \mathrm{~mm}$ e as cortinas laterais da estufa foram fechadas; neste caso, foi notório o efeito da elevação da URmi na demanda hídrica atmosférica dentro da estufa, visto pela redução da Epi, a qual, por ser um processo físico, respondeu mais rapidamente à alteração na condição meteorológica. A ETm não alcançou a mesma magnitude da Epi (Figura 3A), sobretudo por ser regida por mecanismos biológicos de resistência estomática, além de físicos.

Os menores valores da URmi ocorreram no período final do experimento, após os 80 DAT com a elevação da temperatura do ar e da disponibilidade de radiação solar (Figura 2B). Esta condição contribuiu para as maiores diferenças de déficit de saturação do ar nos dois ambientes, com os maiores valores de déficit de saturação do ar encontrados no ambiente interno, neste período.

Do ponto de vista do crescimento das plantas de pimentão a redução na ETm pode, em parte, ser explicada pelo reduzido IAF. Dalmago et al. (2003) encontraram um IAF de até 2,5 para o pimentão cultivado também em estufa plástica, valor consideravelmente superior ao encontrado, o qual alcançou o valor máximo de 1,5 somente no final do experimento, aos 120 DAT (Figura 3A) não contribuindo, de forma gradual, para aumentar a área transpirante desde a fase inicial; outra causa pode ter sido a influência do "mulching" plástico sobre a superfície do solo nos camalhões da bordadura e nos lisímetros, reduzindo também as perdas de água por evapora- ção, que seria computada como ETm (Xie et al., 2005; Medeiros et al., 2005; Wang et al., 2009); desta forma, foi determinada uma ETm menor e, portanto, o erro em relação ao valor absoluto de ETm foi maior.

Após os 60 DAT verificou-se aumento progressivo da ETm (Figura 3A) em resposta ao aumento do fluxo de radiação solar global incidente e do comprimento do dia que determinaram um período maior de tempo em que a estufa permaneceu aberta, facilitando a saída do vapor d'água do seu interior.

O valor máximo de ETm (2,66 mm), se deu aos 106 DAT, coincidindo com valores também elevados de radiação solar e velocidade do vento a $2 \mathrm{~m}$, que atingiu $0,74 \mathrm{~m} \mathrm{~s}^{-1}$, aumentando a retirada de vapor d'água do interior da estufa e, em conseqüência, elevando o déficit de saturação do ar nesse ambiente.

A relação da ETm com a Epi no início do ciclo, quando as plantas se encontravam com um IAF menor que 1,0, não foi satisfatória (Figura 3A); neste período, as condições ambientais favoreceram a elevação da demanda hídrica atmosférica e da evaporação no Piche, já que neste instrumento a perda de água está condicionada, basicamente, pelo poder evaporante do ar; contudo, em função da pequena área foliar transpirante, a ETm não alcançou a magnitude da Epi, o $\mathrm{R}^{2}$ da regressão linear entre a ETm e o IAF para todo o período experimental foi elevado $\left(\mathrm{R}^{2}=0,81\right)$, demonstrando a grande influência do IAF na transpiração das plantas (Figura 3B).

Apresentam-se, na Tabela 1, os 43 modelos de regressão linear para estimativa da ETm, selecionados a partir de um $\mathrm{R}^{2}$ maior do que 0,80 , com os respectivos coeficientes de determinação e coeficientes angulares significativos pelo teste $\mathrm{t} a$ $5 \%$ de probabilidade de erro.

A primeira constatação, a qual está em concordância com Heldwein et al. (2004), é de que a ETm em estufa plástica pode ser estimada a partir da simples medição no interior da estufa de variáveis meteorológicas e fenométricas, com a possibilidade de se usar as leituras meteorológicas medidas em determinados horários fixos.

Nos modelos 19 (IAF), 22 (NF) e 27 (AP), observa-se que a não inclusão de uma terceira variável independente resulta em valores similares de $\mathrm{R}^{2}$ em relação aos dos modelos com essas três variáveis fenométricas. Os modelos 19, 22 e 27 são os mais simples e a AP é a variável fenométrica de mais fácil determinação pelo produtor rural.

A inclusão de uma variável meteorológica adicional medida em horário fixo além da Epi proporcionou, em geral, um pequeno aumento no valor de $\mathrm{R}^{2}$, sendo D9, D15 e T9 as variáveis que mais melhoraram o ajuste do modelo (modelos 1 a 8 , exceto o modelo 7) seguidos, na ordem, pelas variáveis T15, Tmi, Tmax e Tmin.

A inclusão da amplitude térmica diária (AT), juntamente com a Epi, não proporcionou melhora de ajuste nos modelos, conforme a comparação dos valores de $\mathrm{R}^{2}$ entre os modelos 19 e 20,22 e 23 e 26 e 27 (Tabela 1). A AT não melhorou as estimativas da ETm quando incluída no modelo com Epi, provavelmente por sua dependência da Rgi e também porque ambas têm correlação com o déficit de saturação do ar sendo, ainda, a Rgi e o Di as duas variáveis que mais afetam a evaporação (Heldwein et al., 2001) e a ETm em estufas (Dal- 
Tabela 1. Modelos de regressão para estimativa da evapotranspiração máxima (ETm) do pimentão cultivado em estufa plástica, na primavera em 2005

\begin{tabular}{|c|c|c|}
\hline $\mathrm{N}^{0}$ & Modelos de regressão* & $\mathbf{R}^{2}$ \\
\hline & $n=-0,29744+0,82366 \mathrm{IAF}+0,10708 \mathrm{Epi}+0,05733 \mathrm{Dg}$ & 0,918 \\
\hline & $n=-0,56911+0,02077 \mathrm{AP}+0,10623 \mathrm{Epi}+0,06284 \mathrm{Dg}$ & 917 \\
\hline $3 \mathrm{E}$ & $=-0,35326+0,01206 \mathrm{NF}+0,11522$ & 0,913 \\
\hline & $n=-0,31905+0,85739$ IAF $+0,1164 E p i+0,01902 D 15$ & 0,907 \\
\hline & $=-0,60319+0,02157 \mathrm{AP}+0,11799 \mathrm{Epi}+0,02082 \mathrm{D} 15$ & 0,903 \\
\hline $6 \mathrm{E}$ & $=-0,37622+0,01255 \mathrm{NF}+0,13070 \mathrm{Epi}+0,01642 \mathrm{D} 15$ & 0,899 \\
\hline & $n=-0,79051+0,01108 \mathrm{NF}+0,14259 \mathrm{Epi}+0,03038 \mathrm{~T} 9$ & 0,896 \\
\hline & $=-0,71074+0,75993$ IAF $+0,14540 E p i+0,02824 T 9$ & 0,895 \\
\hline $9 \mathrm{E}$ & $=-0,66911+0,83638 \mathrm{IAF}+\mathrm{C}$ & 0,892 \\
\hline $10 \mathrm{E}$ & $n=-0,76200+0,79019 \mathrm{IAF}+0,16794 \mathrm{Epi}+0,02622 \mathrm{Tmi}$ & 0,892 \\
\hline $11 \mathrm{E}$ & $=-0,81037+0,01163 \mathrm{NF}+0,17001 E \mathrm{pi}+0,02596 \mathrm{Tmi}$ & 0,891 \\
\hline $12 \mathrm{E}$ & $=-0,68034+0,084$ & 0,891 \\
\hline $13 \mathrm{E}$ & $n=-0,62082+0,794671 \mathrm{AF}+0,20232 \mathrm{Epi}+0,02217 \mathrm{Tmin}$ & 0,890 \\
\hline $14 \mathrm{E}$ & $=-0,71827+0,01231 \mathrm{NF}+0,147897$ & 0,890 \\
\hline $15 \mathrm{E}$ & $=-0,73425+0,0123$ & 0,889 \\
\hline $16 \mathrm{E}$ & $n=-0,66876+0,01170 N F+0,2039$ & 0,888 \\
\hline $17 \mathrm{E}$ & $=-0,96339+0,01855 A P+0,15529$ & 0,885 \\
\hline $18 \mathrm{E}$ & $=-1,03757+0,01960 A P+0,177$ & 0,884 \\
\hline $19 \mathrm{E}$ & $=-0,32562+0,9156$ & 0,883 \\
\hline $20 \mathrm{E}$ & $=-0,36964+0,92447 \mid \mathrm{AF}+0,16965$ & 0,883 \\
\hline $21 \mathrm{E}$ & $=-0,95469+0,02086 \mathrm{AP}+0,15407$ & 0,883 \\
\hline $22 \mathrm{E}$ & $-0,38667+0,013$ & 0,882 \\
\hline & $-0,43273+0,01360 N F+0,1709$ & 0,882 \\
\hline $24 \mathrm{E}$ & $=-0,96524+0,021$ & 0,881 \\
\hline $25 \mathrm{E}$ & $-0,89767+0,01966 \mathrm{AP}+0,213$ & 0,881 \\
\hline $26 \mathrm{E}$ & $=-0,67250+0,02317 A P+0,1817$ & 0,881 \\
\hline $27 \mathrm{E}$ & $=-0,62825+0,02296 \mathrm{AP}+$ & 0,881 \\
\hline $28 \mathrm{E}$ & AP0,5(-0,02893+0,00129NF $+0,02065 E p i+0,0048$ & 0,880 \\
\hline & AP0,5(-0,09712 + 0,00111NF +0,02431Epi $+0,0040$ & 0,880 \\
\hline $30 \mathrm{E}$ & AP0,5(-0,02844t+ & 0,874 \\
\hline $31 \mathrm{E}$ & AP0,5(-0,08132+0,00108NF +0,02990Epi $+0,00387$ Tmin $)$ & 0,873 \\
\hline $32 \mathrm{E}$ & $=A P 0,5(-0,08035+0,00124 N F+0,02187 \mathrm{Epi}+0,00251 \mathrm{Tmax})$ & 0,872 \\
\hline & AP0,5(-0,03296 +0,00139NF +0,02599Epi $+0,0004$ & 0,86 \\
\hline $34 \mathrm{E}$ & $=A P 0,5(-0,02985+0,00138 \mathrm{NF}+0,02729 E \mathrm{Epi})$ & 0,859 \\
\hline & $=N F 0,5(-0,07001+0,00131 A P+0,02599 E p i+0,0031$ & 0,856 \\
\hline $36 \mathrm{E}$ & NF0,5(-0,07119 + 0,00153AP $+0,01953 \mathrm{Epi}+0,00204 \mathrm{Tmax})$ & 0,852 \\
\hline $37 \mathrm{E}$ & $=$ NF0,5(-0,03226 +0,00173AP +0,02400Epi) & 0,842 \\
\hline & NF0,5(-0,03466 +0,00174AP +0,02305 & ) 0,84 \\
\hline $39 \mathrm{E}$ & IAF0,5(-0,07999+0,00877AP + 0,1674Epi + 0,04310D9) & 0,819 \\
\hline $40 \mathrm{E}$ & IAF0,5(-0,61415+0,00297NF $+0,19116 \mathrm{Epi}+0,03997 \mathrm{Tmi})$ & 0,818 \\
\hline & IAF0,5(-0,45077+0,00321NF +0,24407Epi $+0,03391 T$ min $)$ & 0,812 \\
\hline & IAF0,5(-0,51094+0,0055AP $+0,24587 E p i+0,03407$ Tmin $)$ & 0,812 \\
\hline $43 \mathrm{E}$ & $=\mid$ AF0,5(-0,60405+0,00631AP + 0,19956Epi + 0,0322Tmi) & 0,810 \\
\hline
\end{tabular}

*Todos os coeficientes angulares são significativos pelo teste t a $5 \%$ de probabilidade de erro; $\mathrm{R}^{2}$ - coeficiente de determinação, Epi evaporação no evaporímetro de Piche $\left(\mathrm{mm} \mathrm{dia}^{-1}\right)$; AP - altura de plantas; IAF - índice de área foliar; NF - número de folhas; D9, D15 déficit de saturação do ar interno $(\mathrm{hPa})$ às 9 e $15 \mathrm{~h}$ respectivamente; Tmi, Tmin, Tmax - temperatura média, mínima e máxima do ar $\left({ }^{\circ} \mathrm{C}\right)$ respectivamente; T9, T15 - temperatura do ar às 9 e $15 \mathrm{~h}\left({ }^{\circ} \mathrm{C}\right)$, respectivamente

mago et al., 2006). Portanto, seria lógico esperar que um modelo que inclua somente a variável AT, ao invés de Epi, possa também ser suficientemente preciso para estimar a ETm mas, provavelmente, em um mesmo modelo não poderiam ser utilizadas essas duas variáveis conjuntamente (AT e Epi).
$\mathrm{O}$ cômputo da ETm por unidade de $\mathrm{AP}, \mathrm{AP}^{0,5}, \mathrm{NF}, \mathrm{NF}^{0,5}$, IAF ou IAF ${ }^{0,5}$, também não melhorou o $\mathrm{R}^{2}$ (modelos 28 a 43) (Tabela 1); muito pelo contrário, na grande maioria dos modelos houve diminuição do valor do $\mathrm{R}^{2}$ resultando, inclusive, na eliminação de um grande número dos mesmos por não alcançarem valor de $\mathrm{R}^{2}$ maior do que 0,80 .

Estatísticas do teste dos modelos realizado com os dados coletados no experimento de 2006, isto é, diferentes daqueles de 2005 utilizados na obtenção dos modelos, são apresentadas na Tabela 2. Verifica-se que a utilização do IAF resulta em maior estabilidade dos modelos já que os 12 maiores valores de índice de confiança "c" foram obtidos nos modelos que incluíram o IAF $(39,43,40,42,41,9,12,1,20,8,4$ e 19), cujos desempenhos foram ótimo e muito bom, conforme a escala de Camargo \& Sentelhas (1997) (Tabela 2).

Em geral, o uso do NF como variável fenométrica resultou nos menores valores de "c", com esses modelos classificados, na sua maioria, apenas como bons. A AP, associada ou não ao IAF ou ao NF resultou, comumente, em desempenho intermediário aos encontrados com o IAF e NF, porém todos integraram a classe de modelos muito bons.

Ao se utilizar Epi e IAF sem transformação por unidade de raiz quadrada, a inclusão de uma variável meteorológica adicional, como T15, Tmax, D9, AT, T9 e D15, nesta ordem, aumentou o índice de confiança "c" em no máximo 0,02. Aumento de "c" em 0,029 e 0,058, respectivamente, somente foi obtido quando se utilizou a ETm por unidade de raiz quadrada do IAF (ETm IAF ${ }^{-0,5}$ ), NF e Tmi (modelo 40), ou ETm IAF0,5 , NF e Tmin (modelo 41). Obteve-se incremento similar quando se utilizou nos modelos $\mathrm{ETm}_{\mathrm{IAF}}^{-0,5}$ e AP, além de D9 (modelo 39), Tmin (modelo 42) ou Tmi ( modelo 43).

A inclusão apenas de Tmi além de IAF (sem transformação) e Epi (modelo 10), diminuiu o valor de "c" em 0,052, que ficou no limite inferior da classe de desempenho muito bom enquanto a inclusão de Tmin resultou em uma diminuição de 0,012 no valor do índice de confiança "c"; portanto, quando se dispõe de evaporímetro de Piche e termômetros de máxima e de mínima, ao se utilizar o IAF como única variável fenométrica deve-se optar pelo modelo 12.

Quando a AP foi usada sem transformação por raiz quadrada, junto de Epi (modelo 27), a inclusão de T15 proporcionou melhora de 0,026 no valor de "c", enquanto a inclusão de Tmax (modelo 24), D9 (modelo 2), Tmi (modelo 18) e AT, resultou em apenas uma pequena melhora no valor do índice de confiança "c" (Tabela 2).

A inclusão de Tmin ou D15 piorou o desempenho no teste dos modelos 25 e 5 , respectivamente, em relação ao modelo 27; a Tmin associada a AP confirma a resposta negativa também apresentada por esta variável quando incluída em modelos, juntamente com o IAF ou NF, permitindo inferir que a Tmin deve ser utilizada apenas quando os modelos incluem valores transformados das variáveis fenométricas $\left(\mathrm{IAF}^{0,5}, \mathrm{AP}^{0,5}\right.$, $\mathrm{NF}^{0,5}$ ), visto pelo teste dos modelos 31,35 e 42 . A utilização do déficit de saturação do ar, conforme modelos 1 a 6 , mostrou-se eficiente apenas quando associada ao IAF (modelos 1 e 4) ou à AP; neste caso, para as duas varáveis fenométricas a utilização de D9 mostrou-se mais adequada que D15, possivelmente porque, em dias frios, em especial naqueles 
Tabela 2. Avaliação dos modelos para estimativa da evapotranspiração máxima (ETm) do pimentão cultivado em estufa plástica através da regressão entre os valores medidos na primavera de 2005 e os estimados com os dados da primavera de 2006

\begin{tabular}{ccccccc}
\hline № & RMSE & $\mathbf{R}^{2}$ & $\mathbf{r}$ & $\mathbf{d}$ & $\mathbf{C}$ & Desempenho* \\
1 & 0,2370 & 0,836 & 0,903 & 0,918 & 0,828 & $\mathrm{MB}$ \\
2 & 0,2459 & 0,789 & 0,877 & 0,914 & 0,802 & $\mathrm{MB}$ \\
3 & 0,3165 & 0,764 & 0,863 & 0,831 & 0,717 & $\mathrm{~B}$ \\
4 & 0,2379 & 0,818 & 0,893 & 0,918 & 0,819 & $\mathrm{MB}$ \\
5 & 0,3051 & 0,783 & 0,874 & 0,868 & 0,759 & $\mathrm{~B}$ \\
6 & 0,3142 & 0,741 & 0,850 & 0,834 & 0,709 & $\mathrm{~B}$ \\
7 & 0,2881 & 0,758 & 0,860 & 0,863 & 0,742 & $\mathrm{~B}$ \\
8 & 0,2334 & 0,817 & 0,892 & 0,921 & 0,822 & $\mathrm{MB}$ \\
9 & 0,2262 & 0,831 & 0,900 & 0,926 & 0,834 & $\mathrm{MB}$ \\
10 & 0,2833 & 0,762 & 0,862 & 0,885 & 0,762 & $\mathrm{MB}$ \\
11 & 0,2883 & 0,749 & 0,854 & 0,863 & 0,738 & $\mathrm{~B}$ \\
12 & 0,2281 & 0,824 & 0,896 & 0,925 & 0,829 & $\mathrm{MB}$ \\
13 & 0,2426 & 0,787 & 0,876 & 0,916 & 0,802 & $\mathrm{MB}$ \\
14 & 0,2884 & 0,779 & 0,871 & 0,861 & 0,751 & $\mathrm{~B}$ \\
15 & 0,2881 & 0,769 & 0,866 & 0,862 & 0,747 & $\mathrm{~B}$ \\
16 & 0,2971 & 0,716 & 0,836 & 0,856 & 0,715 & $\mathrm{~B}$ \\
17 & 0,2469 & 0,776 & 0,870 & 0,912 & 0,794 & $\mathrm{MB}$ \\
18 & 0,2449 & 0,780 & 0,872 & 0,915 & 0,798 & $\mathrm{MB}$ \\
19 & 0,2353 & 0,801 & 0,884 & 0,921 & 0,814 & $\mathrm{MB}$ \\
20 & 0,2307 & 0,813 & 0,890 & 0,924 & 0,823 & $\mathrm{MB}$ \\
21 & 0,2365 & 0,797 & 0,882 & 0,921 & 0,812 & $\mathrm{MB}$ \\
22 & 0,3040 & 0,732 & 0,845 & 0,847 & 0,715 & $\mathrm{~B}$ \\
23 & 0,3006 & 0,751 & 0,856 & 0,849 & 0,727 & $\mathrm{~B}$ \\
24 & 0,2387 & 0,792 & 0,878 & 0,920 & 0,808 & $\mathrm{MB}$ \\
25 & 0,2547 & 0,753 & 0,857 & 0,909 & 0,778 & $\mathrm{MB}$ \\
26 & 0,2446 & 0,770 & 0,866 & 0,917 & 0,795 & $\mathrm{MB}$ \\
27 & 0,2494 & 0,758 & 0,860 & 0,914 & 0,786 & $\mathrm{MB}$ \\
28 & 0,2889 & 0,841 & 0,902 & 0,861 & 0,777 & $\mathrm{MB}$ \\
29 & 0,2583 & 0,827 & 0,894 & 0,895 & 0,800 & $\mathrm{MB}$ \\
30 & 0,2910 & 0,822 & 0,891 & 0,860 & 0,767 & $\mathrm{MB}$ \\
31 & 0,2651 & 0,800 & 0,879 & 0,890 & 0,782 & $\mathrm{MB}$ \\
32 & 0,2629 & 0,840 & 0,901 & 0,889 & 0,801 & $\mathrm{MB}$ \\
33 & 0,2790 & 0,820 & 0,890 & 0,873 & 0,777 & $\mathrm{MB}$ \\
34 & 0,2803 & 0,814 & 0,887 & 0,872 & 0,773 & $\mathrm{MB}$ \\
35 & 0,2685 & 0,831 & 0,896 & 0,887 & 0,795 & $\mathrm{MB}$ \\
36 & 0,2632 & 0,856 & 0,909 & 0,891 & 0,810 & $\mathrm{MB}$ \\
37 & 0,2707 & 0,838 & 0,900 & 0,884 & 0,796 & $\mathrm{MB}$ \\
38 & 0,2701 & 0,842 & 0,902 & 0,885 & 0,798 & $\mathrm{MB}$ \\
39 & 0,2096 & 0,874 & 0,923 & 0,945 & 0,872 & 0 \\
40 & 0,2087 & 0,853 & 0,912 & 0,946 & 0,862 & 0 \\
41 & 0,2280 & 0,834 & 0,902 & 0,935 & 0,843 & $\mathrm{MB}$ \\
42 & 0,2118 & 0,839 & 0,904 & 0,946 & 0,856 & $\mathrm{MB}$ \\
\hline 3 & 0,2011 & 0,858 & 0,915 & 0,951 & 0,870 & 0 \\
\hline
\end{tabular}

*Desempenho conforme Camargo \& Sentelhas (1997); RMSE - raiz do quadrado médio do erro; $\mathrm{R}^{2}$ - coeficiente de determinação da curva entre valores observados e estimados; r - coeficiente de correlação; d coeficiente de Willmott; c - índice de confiança de Camargo \& Sentelhas (1997)

com previsão de geada, a estufa foi fechada antes das $15 \mathrm{~h}$ para armazenar calor, o que alterou o valor de D15 em relação à média diária do déficit de saturação do ar, cujo efeito sobre a ETm é mais real que o valor pontual das $15 \mathrm{~h}$.

\section{CONCLUSÕES}

1. A evapotranspiração máxima do pimentão pode ser estimada com precisão aceitável para quantificar a água utilizada na irrigação diária em estufa plástica.

2. A inclusão de uma variável meteorológica adicional proporcionou, em geral, uma pequena melhora no ajuste e no desempenho dos modelos.

3. O uso do índice de área foliar resulta em maior estabilidade dos modelos de estimativa da evapotranspiração máxima do pimentão em relação às variáveis fenométricas número de folhas e altura de plantas.

4. A altura de plantas apresenta desempenho intermediário ao proporcionado pelo índice de área foliar e número de folhas, na estimativa da evapotranspiração máxima do pimentão.

5. Quando associado ao índice de área foliar ou à altura de plantas, o déficit de saturação do ar medido às $9 \mathrm{~h}$, mostrou-se mais adequado que o déficit de saturação do ar medido às $15 \mathrm{~h}$, para a estimativa da evapotranspiração máxima do pimentão.

\section{AGRADECIMENTOS}

À Coordenação de Aperfeiçoamento de Pessoal de Nível Superior (CAPES) pela Bolsa de Mestrado concedida a Carina R. Pivetta, Ivan C. Maldaner e Sidinei Z. Radons, e ao Conselho Nacional de Desenvolvimento Científico e Tecnológico (CNPq) pela Bolsa de Produtividade em Pesquisa concedida a Arno B. Heldwein.

\section{LITERATURA CITADA}

Andriolo, J. L. Fisiologia das culturas protegidas. Santa Maria: UFSM, 1999. 142p.

Araújo, J. S.; Andrade, A. P. de; Ramalho, C. I.; Azevedo, C. A. V. de. Características de frutos de pimentão cultivado em ambiente protegido sob doses de nitrogênio via fertirrigação. Revista Brasileira de Engenharia Agrícola e Ambiental, v.13, n.2, p. 152-157, 2009.

Beckmann, M. Z.; Duarte, G. R. B.; Paula, V. A. de; Mendez, M. E. G.; Peil, R. M. N. Radiação solar em ambiente protegido cultivado com tomateiro nas estações verão-outono do Rio Grande do Sul. Ciência Rural, v.36, n.1, p.86-92, 2006.

Bonachella, S.; Gonzalez, M. A.; Fernandéz, M. D. Irrigation scheduling of plastic greenhouse vegetable crops based on historical weather data. Irrigation Science, v.25, p.25-62, 2006.

Buriol, G. A.; Lago, I.; Heldwein, A. B.; Schneider, F. M.; Andriolo, G. L. Disponibilidade de radiação solar para hortaliças cultivadas em ambiente protegido no período invernal no Estado do Rio Grande do Sul. Revista Brasileira de Agrometeorologia, v.13, n.1, p.21-26, 2005.

Camargo, A. P. Sentelhas, P. C. Avaliação do desempenho de diferentes métodos de estimativa da evapotranspiração potencial no Estado de São Paulo. Revista Brasileira de Agrometeorologia, v.5, n.1, p.89-97, 1997. 
Cardoso, L. S; Bergamaschi, H.; Comiran, F.; Chavarria, G.; Marodin, G. A. B.; Dalmago, G. A.; Santos, H. P. dos; Mandelli, F. Alterações micrometeorológicas em vinhedos pelo uso de coberturas de plástico. Pesquisa Agropecuária Brasileira, v.43, n.4, p.441-447, 2008.

Chavarria, G.; Santos, H. P.; Felippeto, J.; Marodin, G. A. B.; Bergamaschi, H.; Cardoso, L. S.; Fialho, F. B. Relações hídricas e trocas gasosas em vinhedo sob cobertura plástica. Revista Brasileira de Fruticultura, v.30, n.4, p.1022-1029, 2008.

CQFS - Comissão de Química e Fertilidade do Solo. Recomendação de adubação e calagem para os estados do Rio Grande do Sul e Santa Catarina. Porto Alegre: SBCS/Núcleo Regional Sul/UFRGS, 2004. 400p.

Dalmago, G. A. Evapotranspiração máxima e coeficiente da cultura do pimentão em estufa plástica. Santa Maria: UFSM. 2001. 165p. Dissertação Mestrado

Dalmago, G. A.; Heldwein, A. B.; Buriol, G. A.; Luzza, G.; Tazzo, I. F.; Trentin, G. Evapotranspiração máxima e coeficiente de cultura do pimentão em estufa plástica. Revista Brasileira de Agrometeorologia, v.11, n.1, p.33-41, 2003.

Dalmago, G. A.; Heldwein, A. B.; Nied, A. H.; Grimm, E. L.; Pivetta, C. R. Evapotranspiração máxima da cultura de pimentão em estufa plástica em função da radiação solar, da temperatura, da umidade relativa e do déficit de saturação do ar. Ciência Rural, v.36, n.3, p.785-792, 2006.

Heldwein, A. B.; Dalmago, G. A.; Streck, L.; Tazzo, I. F.; Trentin, G. Utilização do evaporímetro de Piche exposto á radiação solar para estimar a evapotranspiração máxima do pimentão em estufa plástica. Revista Brasileira de Agrometeorologia, v.9, n.2, p.213-217, 2001.

Heldwein, A. B.; Streck, L.; Schneider, F. M.; Grimm, E. L.; Nied, A. H.; Tazzo, I. F. Modelos para a estimativa da evapotranspiração máxima da abóbora italiana em estufa plástica. Revista Brasileira de Agrometeorologia, v.12, n.1, p.75-86, 2004.

Lorentz, L. H.; Lúcio, A. D.; Heldwein, A. B.; Souza, M. F.; Mello, R. M. Estimativa da amostragem para pimentão em estufa plástica. Horticultura Brasileira, v.20, n.2, 2002. 4p.

Medeiros, F. A. S. B. de; Medeiros, J. F. de; Silva, M. C. de C.; Alves, L. P.; Souza, T. H. de; Levien, S. L. A. Necessidade hídrica do meloeiro irrigado com água de diferentes salinidades e cultivado com ou sem cobertura do solo. Revista Brasileira de Engenharia Agrícola e Ambiental, v.9, n.2, p.234-238, 2005.
Orgaz, F.; Fernádez, M. D.; Bonachela, S., Gallardo, M.; Fereres, E. Evapotranspiration of horticultural crops in an unheated plastic greenhouse. Agricultural Water Management, v.72, n.2, p.81-96, 2005.

Pereira, A. R.; Nova, N. A. V.; Sediyama, G. C. Evapo(transpi)ração. Piracicaba: ESALQ, 1997. 183p.

Pivetta, C. R. Evapotranspiração máxima do tomate e do pimentão em estufa plástica em função da evaporação no "Piche" e outras variáveis meteorológicas e fenométricas. Santa Maria: UFSM. 2007. 83p. Dissertação Mestrado

Reis, L. S.; Souza, J. L. de; Azevedo, C. A. V. de. Evapotranspiração e coeficiente de cultivo do tomate caqui cultivado em ambiente protegido. Revista Brasileira de Engenharia Agrícola e Ambiental, v.13, n.3, p.289-296, 2009.

Righi, E. Z.; Angelocci, L. R.; Buriol, G. A.; Heldwein, A. B. Transpiração do tomateiro cultivado em estufa plástica e suas relações com a radiação solar e déficit de saturação do ar. Revista Brasileira de Agrometeorologia, v.10, n.1, p.9-18, 2002.

Tazzo, I. F.; Heldwein, A. B.; Grimm, E. L.; Maass, G. F.; Pivetta, C. R. Evapotranspiração máxima e coeficiente de cultura para o pimentão cultivado em estufa plástica na primavera. Revista Brasileira de Agrometeorologia, v.2, n.2, p.275-281, 2004.

Valandro, J.; Andriolo, J. L.; Buriol, G. A. Dispositivo lisimétrico simples para determinar a transpiração das hortaliças cultivadas fora do solo. Revista Brasileira de Agrometeorologia, v.7, n.2, p.189-193, 1999.

Valandro, J.; Buriol, G. A.; Andriolo, J. L.; Heldwein, A. B. Transpiração do tomateiro cultivado fora do solo em estufa plástica e sua relação com os elementos meteorológicos. Ciência Rural, v.37, n.6, p.1593-1600. 2007.

Vasquez, M. A. N.; Folegatti, M. V.; Dias, N. S.; Silva, C. R. da. Efeito do ambiente protegido cultivado com melão sobre os elementos meteorológicos e sua relação com as condições externas. Engenharia Agrícola, v.25, n.1, p.137-143, 2005.

Wang, F. X.; Feng, S. Y.; Hou, X. Y., Kang, S. Z. Potato growth with and without plastic mulch in two typical regions of Northern China. Field Crops Research, v.110, n.2, p.123-129, 2009.

Xie, Z.; Wang, Y.; Li, F. Effect of plastic mulching on soil water use and spring wheat yeld in arid region of northwest China. Agricultural Water Manegement, v.75, n.1, p.71-83, 2005. 